\title{
Wide-Area Robust Decentralized Coordinated Control of HVDC Power System Based on Polytopic System Theory
}

\author{
Shiyun Xu, ${ }^{1}$ Huadong Sun, ${ }^{1}$ Baiqing Li, ${ }^{1}$ Guangquan Bu, ${ }^{1}$ Jun Yi, ${ }^{1}$ Jian Zhang, \\ Bing Zhao, ${ }^{1}$ and Zhanming Chen ${ }^{2}$ \\ ${ }^{1}$ China Electric Power Research Institute, Beijing 100192, China \\ ${ }^{2}$ Renmin University of China, Beijing 100872, China \\ Correspondence should be addressed to Zhanming Chen; chenzhanming@pku.edu.cn
}

Received 9 January 2015; Accepted 10 March 2015

Academic Editor: Xinggang Yan

Copyright (C) 2015 Shiyun Xu et al. This is an open access article distributed under the Creative Commons Attribution License, which permits unrestricted use, distribution, and reproduction in any medium, provided the original work is properly cited.

The present study proposes a hierarchical wide-area decentralized coordinated control framework for HVDC power system that is robust to multiple operating conditions. The upper level wide-area coordinated controller is designed in the form of dynamic output feedback control that coordinates the lower level HVDC supplementary controller, PSS, and SVC. In order to enhance the robustness of the designed controller under various operating conditions, the polytopic model is introduced such that the closed-loop control system can be operated under strong damping mode in virtue of the stability criterion based on damping ratio. Simulation results demonstrate that the proposed controller design algorithm is capable of enhancing the system damping over four different conditions.

\section{Introduction}

With the construction of large-volume transmission projects with extensive interconnected lines in China, the power grid structure has become even more complex, and the continuous and increasing demand of electrical energy consumption has greatly influenced the power system performance. The large interconnected power systems can improve the use of the existing generation resources, and moreover they allow the cooperation among the interconnected systems in case of faults and to guarantee the power supply in contingency periods [1]. On the other hand, however, if no adequate damping is available, the interarea low-frequency oscillations brought about by such systems may cause operational limitations due to the restrictions in the power transfers across the transmission lines or result in the system separation in certain cases.

Traditionally, power system stabilizer (PSS) is chosen to deal with such problems, whose feedback control loop is commonly composed by local measurements, and moreover the decentralized PSS devices are short of coordination, which cannot eliminate interarea oscillation modes [2]. Due to its economic and unique rapid response characteristics, high voltage DC (HVDC) transmission technique is commonly used as dynamic regulation measures for damping lowfrequency oscillation, improving the power transmission capability of DC/AC hybrid system, and enhancing the AC system transient stability $[3,4]$. Aiming at operating the power system near the stability limits and maintaining dynamic performance at acceptable levels, the design of an additional effective control scheme should be considered. In this regard, DC supplementary control is widely applied in HVDC systems due to its effective enhancement of damping [5]. On the other hand, the operation of HVDC systems demands flexible improvement of power flow distribution, system stability, and transmission capability [6]. Possessing the property of rapid and reliable regulation, flexible AC transmission system (FACTS) devices are implemented to enhance the system stability, and the use of FACTS devices in transmission lines has allowed more effective damping of the interarea oscillations with the addition of supplementary controllers. However, it is pointed out that separated design of PSS, DC supplementary controller, and FACTS damping controller may cause dynamic interactions among them, which results in the instability of the interarea oscillations. 
Since coordinated control can maximize the control effect and, at the same time, avoid possible adverse interaction among existing devices, the research of coordinated control based on wide-area measurement system (WAMS) [7] has achieved satisfying control effects. Aiming at different kinds of control objectives, researchers have been dedicated over the past years to the coordination among PSS, FACTS, and DC supplement controllers based on modern control techniques $[8,9]$. These results reveal the necessity of a coordination procedure, which can remarkably enhance system stability and eliminate mutual influence among equipment.

Power systems undergo changes due to variations in the load, generation, and the transmission network. The variations of operating conditions lead to accompanying changes in the system dynamics, and a well-designed control strategy is guaranteed to perform satisfactorily in the presence of such variations. It is necessary to point out that, however, one of the major drawbacks for most of the existing methods is that the coordinated controller is derived by linearization at the equilibrium point under one particular operating condition. In this regard, the closed-loop control system cannot be guaranteed to remain robust due to its effectiveness to this single equilibrium, and how to meet the demand of operation under multiple cases is still an important issue to be solved.

In order to solve the abovementioned problems, in this study, a wide-area coordinated control strategy among PSS, FACTS damping controllers, and DC supplement controllers applicable to multiple operating conditions in HVDC transmission systems is investigated. In what follows, a wide-area robust decentralized coordinated control (WRDCC) framework with a hierarchical structure is proposed, where the upper level wide-area coordinated controller receives the WAMS measurement signals of HVDC system, and allocates them in the form of coordinated control signal to the lower level generators, DC supplement controllers, and FACTS devices as auxilary input variable of coordinated control. Since not all the model state variables are available for direct measurement in the real system, the coordinated controller is adopted as an output feedback control structure. Due to the simultaneous requirements of robustness, decentralization and coordination of various controllers, the WRDCC algorithm is proposed based on polytopic system theory that is capable of remaining robust to various operating conditions. The decentralized coordinated controller can be conveniently derived by solving three groups of low-dimensional linear matrix inequalities (LMIs) $[10,11]$ through the Matlab control toolbox. Simulation results validated in a 4-machine, 2-area HVDC system demonstrate that the controlled power system successfully runs in strong damping modes in four different operating conditions, and rotor angle and power oscillation are suppressed.

\section{Problem Formulation}

In order to make preparation for the coordination control strategy, in this section, we first introduce the basic formulation of dynamic power system models and then present the WRDCC framework of HVDC power system.

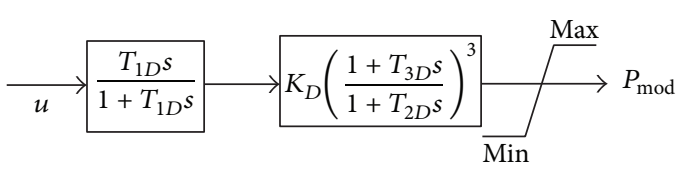

FiguRE 1: HVDC supplementary control.

2.1. HVDC Power System Modelling. For the sake of simplicity, the dynamic devices considered in this study mainly include generators, HVDC supplementary controllers, and FACTS devices.

(1) The generator is represented as a 4-order model equipped with a rapid excitation, whose model can be described as

$$
\begin{gathered}
\dot{\delta}(t)=\omega(t)-\omega_{0}, \\
\dot{\omega}(t)=\frac{\omega_{0}}{H} P_{m}-\frac{D}{H}\left(\omega_{i}(t)-\omega_{0}\right)-\frac{\omega_{0}}{H} P_{e}, \\
\dot{E}_{q}^{\prime}(t)=-\frac{1}{T_{d o}^{\prime}}\left[E_{q}^{\prime}(t)+I_{d}\left(x_{d}-x_{d}^{\prime}\right)\right]+\frac{1}{T_{d o}^{\prime}} V_{f}(t), \\
z(t)=\delta(t)-\delta_{0},
\end{gathered}
$$

where the physical meaning of electrical quantities in (7) can be found in [12].

(2) HVDC supplementary controllers are as follows.

Due to the its economic and unique rapid response characteristics, DC transmission systems can damp lowfrequency oscillation, improve the power transmission capability of DC/AC hybrid system, and enhance the AC system transient stability. The research of HVDC system stability and control neglects the dynamic characteristics of DC lines, and the dynamic variation process of DC power can be described as [7]

$$
\frac{d P_{\mathrm{DC}}}{d t}=\frac{1}{T_{\mathrm{DC}}}\left(-P_{\mathrm{DC}}+P_{\mathrm{DC}, \mathrm{ref}}+P_{\mathrm{mod}}\right)
$$

where $P_{\text {mod }}$ is the DC modulation power, $P_{\mathrm{DC}}$ is the $\mathrm{DC}$ power, $P_{\mathrm{DC} \text {,ref }}$ is the given $\mathrm{DC}$ power reference, and $T_{\mathrm{DC}}$ is the time constant of DC system.

In HVDC systems, the DC line supplementary control parallel to AC lines can eliminate the low frequency of interconnected systems, and its control loop is given in Figure 1, where $T_{1 D}, T_{2 D}$, and $T_{3 D}$ are lead-lag control loop constants and $P_{\text {set }}$ is the modulation limitation. AC system damping can be improved by reasonably chosen DC control signal $u=P_{\text {mod }}$.

(3) Static Var Compensator (SVC) is one of the most widely applied FACTS devices which can maintain voltage stability and, at the same time, improve the system damping. In this study, an SVC is included in the HVDC power system. The block diagram regarding the adopted SVC is given in Figure 2. 


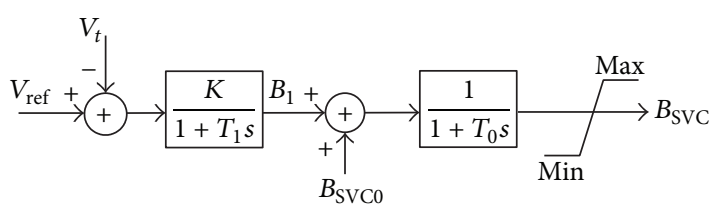

Figure 2: Dynamic model of SVC.

The dynamical model of SVC obtained from the block diagram is described as

$$
\begin{gathered}
\dot{B}_{1}=\frac{1}{T_{1}}\left[-B_{1}+K\left(V_{\mathrm{ref}}-V_{t}\right)\right], \\
\dot{B}_{\mathrm{SVC}}=\frac{1}{T_{0}}\left[B_{1}-B_{\mathrm{SVC}}\right],
\end{gathered}
$$

where $B_{\mathrm{SVC}}$ is the equivalent susceptance output of SVC, $B_{\mathrm{SVC}}$ is the steady-state susceptance of SVC, $B_{1}$ is an intermediate variable, and $K$ is the gain of controller measurement. $T_{1}$ and $T_{0}$ are time constants, $V_{\text {ref }}$ is the reference voltage, and $V_{t}$ is the measurement voltage of the SVC control point.

By integrating equations (1)-(3), we derive the power system model composed by multiple generators, DC compensation controller, and SVC. After linearization around an equilibrium point, the state-space power system model can be represented by a linear time invariant (LTI) model given by a set of linear equations:

$$
\begin{gathered}
\dot{\mathbf{X}}=\mathbf{A X}+\mathbf{B U}, \\
\mathbf{Y}=\mathbf{C X},
\end{gathered}
$$

where $\mathbf{X}$ is the $n$-dimensional state vector, $\mathbf{U}$ is the $p$ dimensional system control input vector, and $\mathbf{Y}$ is the $q$ dimensional system output vector. A, B, C are given system parameter matrices with appropriate dimensions.

\subsection{Decentralized Control Framework Based on WAMS.} When PSS, DC supplementary controller, and FACTS devices are simultaneously equipped in the HVDC power system, the way of coordination should be considered. Commonly speaking, coordinated control among devices/controllers includes decentralized and centralized control. Under decentralized coordinated control, each controller receives the WAMS information and control command individually. The operational status and requirements of other controllers in the area are obtained through information communication. Under the centralized coordinated control, however, WAMS information is normally received through a coordinated controller. The control instruction of each controller can be obtained after calculation. The instruction is then assigned by the coordinated controller to achieve coordinated control. The two methods both have advantages and disadvantages: the former one is more reliable, but the number of devices is large, which makes the coordinated control algorithms for each device and communication lines between devices become complicated; the latter one can achieve global control through coordination control in a better way, but the control

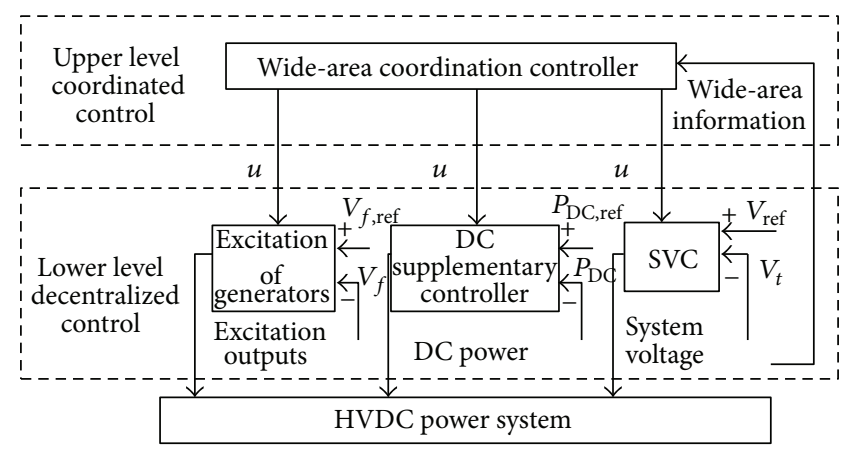

FIGURE 3: WRDCC framework of HVDC system.

speed and accuracy may be affected due to the demand of receiving entire system information.

In this study, decentralized coordinated control is chosen to coordinate PSS, DC supplementary controller, and FACTS devices. In this regard, the WRDCC framework adopts a hierarchical structure, where the upper level wide-area coordinated controller receives the WAMS measurement signals of HVDC system, and allocates them in the form of coordinated control signal $u$ to the lower level generators, DC supplement controllers, and FACTS devices as auxilary input variable of coordinated control. By incorporating the dynamic output feedback control introduced in the previous section, the present study proposes the WRDCC structure shown in Figure 3, where the DC supplement controller and FACTS devices, respectively, given in Figures 1 and 2 are equipped at the bus of DC line of the HVDC system.

\section{Robust Decentralized Coordinated Controller Design Algorithm}

3.1. Polytopic System Model of the Closed-Loop Control System. It can be seen from Figure 3 that since the control input vector $\mathbf{U}$ is derived based on the system output vector $\mathbf{Y}$, the wide-area coordinated controller adopts the dynamic output feedback control strategy for better dynamic characteristics, and accordingly the controller structure is given in

$$
\begin{gathered}
\dot{\mathbf{X}}_{C}=\mathbf{A}_{C} \mathbf{X}_{C}+\mathbf{B}_{C} \mathbf{Y}, \\
\mathbf{U}=\mathbf{C}_{C} \mathbf{X}_{C} .
\end{gathered}
$$

Here, $\mathbf{X}_{C}$ is the $n$-dimensional state vector of controller and $\mathbf{A}_{C}, \mathbf{B}_{C}, \mathbf{C}_{C}$ are parameter matrices to be determined.

The closed-loop controlled system can be derived from (4)-(5) in which

$$
\dot{\tilde{\mathbf{X}}}=\widetilde{\mathbf{A}} \widetilde{\mathbf{X}}
$$

where

$$
\widetilde{\mathbf{X}}=\left[\begin{array}{c}
\mathbf{X} \\
\mathbf{X}_{\mathbf{C}}
\end{array}\right], \quad \widetilde{\mathbf{A}}=\left[\begin{array}{cc}
\mathbf{A} & \mathbf{B C}_{C} \\
\mathbf{B}_{C} \mathbf{C} & \mathbf{A}_{C}
\end{array}\right] .
$$


One of the most commonly used system stability criteria is the Lyapunov method. For LTI system (6), choose a Lyapunov function [13]:

$$
\mathbf{V}(\mathbf{x}, t)=\mathbf{x}^{\mathrm{T}}(t) \mathbf{P} \mathbf{x}(t) .
$$

Based on the Lyapunov direct method, the problem of stabilizing system (1) by output feedback controller (5) can be solved if and only if there exist matrices $\mathbf{A}_{C}, \mathbf{B}_{C}, \mathbf{C}_{C}$, and $\widetilde{\mathbf{P}}$ for system (6) such that the following matrix inequality holds:

$$
\widetilde{\mathbf{A}}^{\mathrm{T}} \widetilde{\mathbf{P}}+\widetilde{\mathbf{P}} \widetilde{\mathbf{A}}<0
$$

In system (6)-(7), the parameter matrix $\widetilde{\mathbf{A}}$ varies followed by the variation of operating condition. If system stability under different operating conditions is satisfied simultaneously, matrix inequality (9) should be guaranteed for each operating condition, which leads to the complicatedness of calculation process. In order to solve the abovementioned problem, the robust damping controller design method proposed in [14] treats the operating condition variation as uncertainties of nominal systems.

Aiming at satisfying the robustness requirements, the polytopic modelling method is introduced in this study. Here, a polytopic model is composed by a series of $p$ typical operating points. More specifically, under the $i$ th operating condition, parameter matrices of system (6) are presented as $\mathbf{A}_{i}, i=1, \ldots, p$, which forms vertices of the polytope. The parameter matrices of state equations under the above $p$ operating conditions compose a set:

$$
\Phi=\left\{\mathbf{A}_{1}, \mathbf{A}_{2}, \ldots, \mathbf{A}_{m}\right\} .
$$

Construct a polytope $\Omega$ whose vertices are composed by elements of set $\Phi$ :

$$
\mathbf{\Omega} \triangleq\left\{\sum_{i=1}^{m} s_{i} \mathbf{A}_{i}, \quad \mathbf{A}_{i} \in \Phi, \sum_{i=1}^{m} s_{i}=1, s_{i} \in \mathbf{R}, s_{i} \geq 0\right\} .
$$

Then, for each vertex system, the closed-loop control system with a dynamic output feedback controller can be written as

$$
\dot{\tilde{\mathbf{X}}}=\widetilde{\mathrm{A}}_{i} \widetilde{\mathbf{X}}
$$

with

$$
\widetilde{\mathbf{X}}=\left[\begin{array}{c}
\mathbf{X} \\
\mathbf{X}_{\mathbf{C}}
\end{array}\right], \quad \widetilde{\mathbf{A}}_{i}=\left[\begin{array}{cc}
\mathbf{A}_{i} & \mathbf{B C}_{C} \\
\mathbf{B}_{C} \mathbf{C} & \mathbf{A}_{C}
\end{array}\right],
$$

where variables are defined the same as in (6)-(7). In this regard, (9) can be interpreted as finding positive definite matrix $\widetilde{\mathbf{P}}$ and appropriate control parameter matrices $\mathbf{A}_{C}, \mathbf{B}_{C}, \mathbf{C}_{C}$ such that the following inequalities hold for $i=$ $1, \ldots, p$ :

$$
\widetilde{\mathbf{A}}_{i}^{\mathrm{T}} \widetilde{\mathbf{P}}+\widetilde{\mathbf{P}} \widetilde{\mathbf{A}}_{i}<0
$$

Based on the polytopic property, matrices $\mathbf{A}_{C}, \mathbf{B}_{C}, \mathbf{C}_{C}$ that satisfy (14) can simultaneously stabilize, not limited to the chosen $p$ vertex systems, all of the linear models included in the polytope. In other words, calculation procedure has been greatly simplified by utilizing the polytopic model.
3.2. Robust Performance Criterion. It is noted that condition (14) only guarantees that system (12) is asymptotically stable. However, power systems may possibly be operated under weak damping modes, which does not meet the practical demand of operation that strong damping mode should be guaranteed. The limited robustness is also one of the major drawbacks for the classical PSS, which derives from the fact that this controller is designed over a single LTI model based on the Lyapunov stability. Generally speaking, the minimal system damping ratio determines its safe operating region. Classical damping controller design, however, cannot guarantee a global minimum damping ratio of the system.

Accordingly, stability criterion based on damping ratio to improve the robustness of the designed controllers should be considered. In this study, the pole placing method [15] is adopted, which can be applied in the damping ratio placement of power systems.

Theorem 1 (see [15]). Let $\theta_{0}=\arccos \zeta_{0}$, for a given minimum damping ratio $\zeta_{\min }$ of closed-loop system (6). If and only if there exists positive definite matrix $\widetilde{\mathbf{P}}$ such that the following matrix inequality

$$
\left[\begin{array}{cc}
\sin \sigma\left(\widetilde{\mathbf{A}}^{\mathrm{T}} \widetilde{\mathbf{P}}+\widetilde{\mathbf{P}} \widetilde{\mathbf{A}}\right) & \cos \sigma\left(\widetilde{\mathbf{A}}^{\mathrm{T}} \widetilde{\mathbf{P}}-\widetilde{\mathbf{P}} \widetilde{\mathbf{A}}\right) \\
* & \sin \sigma\left(\widetilde{\mathbf{A}}^{\mathrm{T}} \widetilde{\mathbf{P}}+\widetilde{\mathbf{P}} \widetilde{\mathbf{A}}\right)
\end{array}\right]<0
$$

holds, then system (6) is said to be asymptotically stable, and meanwhile $\zeta_{\min } \geq \zeta_{0}$ is guaranteed.

Matrix inequality (15) is more appropriate for power system control compared with (9). In power systems, the threshold of damping ratio $\zeta_{0}$ is commonly chosen as 0.03 or 0.05. If and only if the damping ratios of all operation modes are larger than this threshold, the system is said to be operated under a strong damping mode.

However, since the parameter matrix $\widetilde{\mathbf{A}}$ in (15) including unknown controller parameter matrix variables $\mathbf{A}_{C}, \mathbf{B}_{C}$, and $\mathbf{C}_{C}$ is coupled with unknown matrix variable $\widetilde{\mathbf{P}}$, (15) turns out to be a nonlinear matrix inequality, which can be solved by iteration and leads to calculation time consumption and low efficiency.

In order to solve the abovementioned problem, [16] proposes a decoupling method of decentralized coordinated controller design, which transforms (15) into an LMI that is conveniently solvable through Matlab LMI control toolbox. Through expressing $\mathbf{A}_{C}, \mathbf{B}_{C}$, and $\mathbf{C}_{C}$ as diagonal matrices, the controller added to each generator is related to its own input and output. For the sake of space, the following lemma is given for (9).

\section{Lemma 2. Define diagonal matrices}

$$
\widetilde{\mathbf{P}}=\left[\begin{array}{cc}
\mathbf{X} & \mathbf{U} \\
\mathbf{U}^{\mathrm{T}} & \mathbf{X}_{C}
\end{array}\right], \quad \widetilde{\mathbf{P}}^{-1}=\left[\begin{array}{cc}
\mathbf{Y} & \mathbf{V} \\
\mathbf{V}^{\mathrm{T}} & \mathbf{Y}_{C}
\end{array}\right]
$$


and matrix variables $\mathbf{M}=\mathbf{V A}_{C}^{\mathrm{T}} \mathbf{U}^{\mathrm{T}}, \mathbf{P}=\mathbf{Y}^{-1}, \mathbf{F}=\mathbf{U B}_{C}, \mathbf{S}=$ $\mathbf{Y}^{-1} \mathbf{M}, \mathbf{L}=\mathbf{C}_{C} \mathbf{V}^{T}$; then (9) is equivalent to the following LMIs:

$$
\begin{gathered}
{\left[\begin{array}{cc}
\Pi & \mathbf{P A}+\mathbf{A}^{\mathrm{T}} \mathbf{X}+\mathbf{C}_{C}^{\mathrm{T}} \mathbf{B}^{\mathrm{T}} \mathbf{X}+\mathbf{C}^{\mathrm{T}} \mathbf{F}^{\mathrm{T}}+\mathbf{S} \\
* & \mathbf{A}^{\mathrm{T}} \mathbf{X}+\mathbf{X} \mathbf{A}+\mathbf{F} \mathbf{C}+\mathbf{C}^{\mathrm{T}} \mathbf{F}^{\mathrm{T}}
\end{array}\right]<0} \\
\mathbf{A Y}+\mathbf{Y A}^{\mathrm{T}}+\mathbf{B} \mathbf{L}+\mathbf{L}^{\mathrm{T}} \mathbf{B}^{\mathrm{T}}<0
\end{gathered}
$$

where $\Pi=\mathbf{P A}+\mathbf{P B C}_{C}+\mathbf{A}^{\mathrm{T}} \mathbf{P}+\mathbf{C}_{C}^{\mathrm{T}} \mathbf{B}_{C} \mathbf{P}$ and $*$ denotes the symmetric part of the matrix.

In order to design decentralized PSS controller, [17] applies Lemma 2 to matrix inequality (15) and achieves satisfying results. In this study, we use it to the coordinated control algorithm design for HVDC system and, based on the derived results, propose the robust coordinated control algorithm.

Theorem 1 can be easily extended to the polytopic system model shown as below.

Theorem 3. If the minimum damping ratio of system (12) is $\zeta_{\text {min }}$, then, for $\mathbf{A}_{i}, i=1,2, \ldots, p$ in $\Omega$, there is a positive definite symmetric matrix $\widetilde{\mathbf{P}}$ such that the following $p$ matrix inequalities

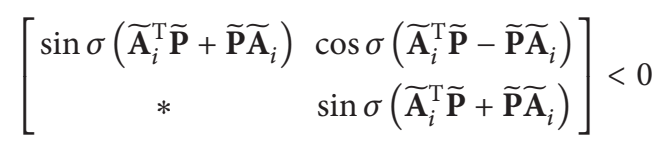

hold; then $\zeta_{\min } \geq \zeta_{0}$, where $\sigma=\arccos \zeta_{0}$.

Proof. It is obvious that, for each vertex system $\mathbf{A}_{i}, i=$ $1,2, \ldots, p$ in $\Omega$, one can arrive at a criterion in the form of (15). Then the damping ratio $\zeta_{\min } \geq \zeta_{0}$ is guaranteed only if the derived $p$ inequalities (18) hold. Moreover, if the $p$ matrix inequalities (18) corresponding to the $p$ vertex systems are satisfied, then the damping ratios of systems included in the polytope all satisfy $\zeta_{\min } \geq \zeta_{0}$.

3.3. WRDCC Algorithm. Choose $p$ typical operating points and linearize the system equations around these points, which obtains parameter matrices of the corresponding state equation $\widetilde{\mathbf{A}}_{i}, \mathbf{B}$, and $\mathbf{C}$ for $i=1, \ldots, p$. Carry out Hankel order reduction [18] and form new state matrix parameters $\mathbf{A}_{i}, i=$ $1, \ldots, p$. Specifically, the WRDCC algorithm comprises the following two steps.

(1) Set damping ratio threshold $\zeta_{0}$ and calculate $\sigma=$ $\arccos \zeta_{0}$. Solve the following LMIs for $i=1,2, \ldots, p$ :

$$
\left[\begin{array}{cc}
\boldsymbol{\Xi}_{11} & \cos \sigma\left(\mathbf{Y A}_{i}^{\mathrm{T}}-\mathbf{A}_{i} \mathbf{Y}+\mathbf{L}^{\mathrm{T}} \mathbf{B}^{\mathrm{T}}-\mathbf{B L}\right) \\
* & \sin \sigma\left(\mathbf{A}_{i} \mathbf{Y}+\mathbf{Y} \mathbf{A}_{i}^{\mathrm{T}}+\mathbf{B L}+\mathbf{L}^{\mathrm{T}} \mathbf{B}^{\mathrm{T}}\right)
\end{array}\right]<0
$$

where $\boldsymbol{\Xi}_{11}=\sin \sigma\left(\mathbf{A}_{i} \mathbf{Y}+\mathbf{Y} \mathbf{A}_{i}^{\mathrm{T}}+\mathbf{B} \mathbf{L}+\mathbf{L}^{\mathrm{T}} \mathbf{B}^{\mathrm{T}}\right)$; then we arrive at positive symmetric matrix variables $\mathbf{Y}>0$ and matrix variable $\mathbf{L}$, and the controller parameter variable $\mathbf{C}_{\mathbf{C}}$ can be obtained by $\mathbf{C}_{C}=\mathbf{L} \mathbf{Y}^{-1}$.

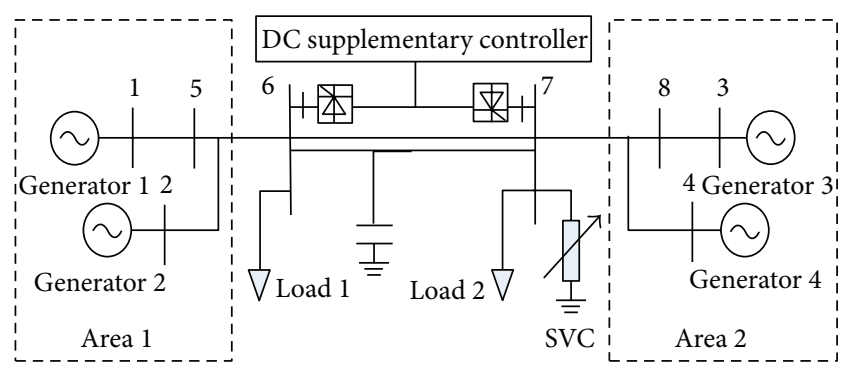

FIgURE 4: A two-area, four-machine HVDC power system.

(2) Let $\mathbf{W}_{i}=\mathbf{A}_{i}+\mathbf{B C}_{C}, i=1,2$ and $p$, and solve the following LMIs:

$$
\left[\begin{array}{ll}
\mathbf{P} & \mathbf{P} \\
\mathbf{P} & \mathbf{X}
\end{array}\right]>0, \quad\left[\begin{array}{cccc}
\boldsymbol{\Theta}_{11} & \boldsymbol{\Theta}_{12} & \boldsymbol{\Theta}_{13} & \boldsymbol{\Theta}_{14} \\
* & \boldsymbol{\Theta}_{22} & \boldsymbol{\Theta}_{14}^{\mathrm{T}} & \boldsymbol{\Theta}_{24} \\
* & * & \boldsymbol{\Theta}_{11} & \boldsymbol{\Theta}_{12} \\
* & * & * & \boldsymbol{\Theta}_{22}
\end{array}\right]<0,
$$

where

$$
\begin{aligned}
& \boldsymbol{\Theta}_{11}=\sin \sigma\left(\mathbf{P W}_{i}+\mathbf{W}_{i}^{\mathrm{T}} \mathbf{P}\right), \\
& \boldsymbol{\Theta}_{12}=\sin \sigma\left(\mathbf{P A}_{i}+\mathbf{W}_{i}^{\mathrm{T}} \mathbf{X}+\mathbf{C}^{\mathrm{T}} \mathbf{F}^{\mathrm{T}}+\mathbf{S}\right), \\
& \boldsymbol{\Theta}_{13}=\cos \sigma\left(\mathbf{W}_{i}^{\mathrm{T}} \mathbf{P}-\mathbf{P} \mathbf{W}_{i}\right), \\
& \boldsymbol{\Theta}_{14}=\cos \sigma\left(-\mathbf{P} \mathbf{A}_{i}+\mathbf{W}_{i}^{\mathrm{T}} \mathbf{X}+\mathbf{C}^{\mathrm{T}} \mathbf{F}^{\mathrm{T}}+\mathbf{S}\right), \\
& \boldsymbol{\Theta}_{22}=\sin \sigma\left(\mathbf{X A}_{i}+\mathbf{W}_{i}^{\mathrm{T}} \mathbf{X}+\mathbf{F} \mathbf{C}+\mathbf{C}^{\mathrm{T}} \mathbf{F}^{\mathrm{T}}\right), \\
& \boldsymbol{\Theta}_{24}=\cos \sigma\left(-\mathbf{X} \mathbf{A}_{i}+\mathbf{A}_{i}^{\mathrm{T}} \mathbf{X}-\mathbf{F C}+\mathbf{C}^{\mathrm{T}} \mathbf{F}^{\mathrm{T}}\right) ;
\end{aligned}
$$

then we get the symmetric matrix variables $\mathbf{P}, \mathbf{X}$ and matrix variables F, $\mathbf{S}$. Controller parameter variables $\mathbf{A}_{C}, \mathbf{B}_{C}$ can be solved through

$$
\begin{array}{cc}
\mathbf{M}=\mathbf{P}^{-1} \mathbf{S}, & \mathbf{A}_{C}=\mathbf{U}^{-1} \mathbf{M}^{\mathrm{T}} \mathbf{P}, \\
\mathbf{U}=\mathbf{P}-\mathbf{X}, & \mathbf{B}_{C}=\mathbf{U}^{-1} \mathbf{F} .
\end{array}
$$

So far, the parameter matrices of the dynamic feedback controller $\mathbf{A}_{C}, \mathbf{B}_{C}$, and $\mathbf{C}_{C}$ are solved.

\section{Numerical Example}

In order to demonstrate the effectiveness of the proposed coordinated control algorithm, consider the following twoarea, four-machine HVDC system equipped with a DC supplementary controller and an SVC shown as in Figure 4, where the detailed system parameters can be referred to [12].

Since the voltage of bus 7 is the lowest, an SVC is equipped to increase voltage, and at the same time a DC supplementary controller is equipped in the bus of interarea system tieline to maintain the stability of power and damp interarea oscillations. The adopted DC supplementary controller and 


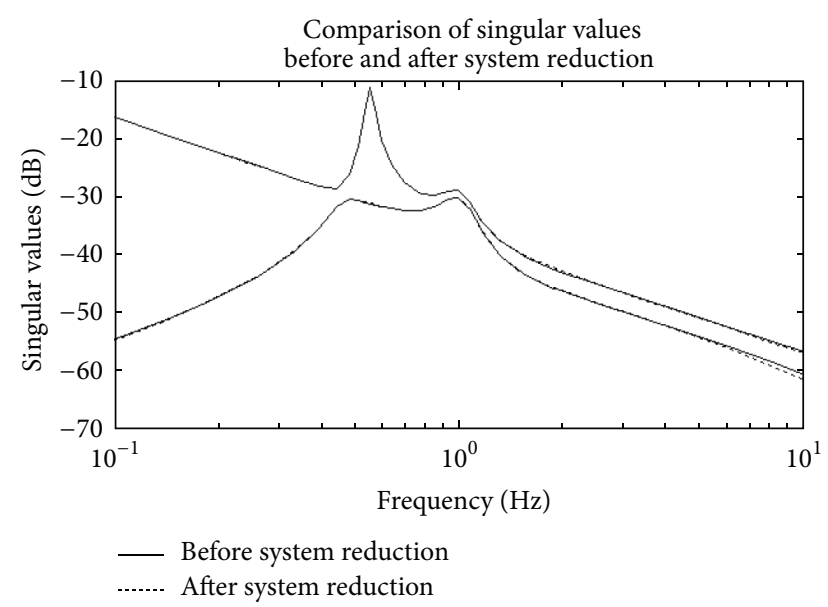

FIGURE 5: Singular values of the nonreduced and reduced systems.

SVC are in the form of dynamical models given in Figures 1 and 2, respectively. Damping ratio threshold is set as $\zeta_{0}=0.1$ in this simulation. Parameters of devices in Figure 4 are given in Table 1.

In virtue of the standard parameter tuning method for SISO controller design [19], the parameters of SVC can be derived as

$$
K=100, \quad T_{1}=0.05, \quad T_{0}=0.01,
$$

while the parameters of DC supplementary controller can be derived as

$$
K_{D}=110, \quad T_{1 D}=0.03, \quad T_{2 D}=0.02 .
$$

In the carried out design, the machine model with excitation regulator has order 3 , the DC power variation with DC supplementary controller model has order 3, SVC model has order 2, and, together with the designed output feedback controller, the entire dynamic linear differential equation of system (6) has order 34 . Solving the 34th-order state equation brings about great difficulties to the calculation of coordinated control algorithm.

In order to speed up calculation, the Hankel reduction method [18] is applied to reduce the system into a 7th-order model. Figure 5 shows the comparison of singular values of the nonreduced and reduced systems. It can be observed from Figure 5 that the two curves almost coincide in a wide range of frequencies.

Based on the derived reduced order system, we design the coordinated controller in virtue of the proposed algorithm in Section 3.3 to coordinate SVC, DC supplementary controller, and PSS, and this can guarantee that the minimum damping ratio satisfies $\zeta_{\min } \geq 10 \%$. Effectiveness of the control strategy is verified in the following operating conditions.

Case 1. Increase Load 1 by $100 \mathrm{MW}$ at $t=0.1 \mathrm{~s}$.

In Cases 2-4, a three-phase short circuit fault occurs in the transmission line between buses 5 and 6 . Denote $\lambda$ by the position of fault, that is, the ratio of length between the fault and bus 5 and length between the fault and bus 6 .
TABle 1: Parameters of devices in Figure 4.

\begin{tabular}{ll}
\hline Devices & Parameters \\
\hline Generator 1 & $x_{d i}=1.569$ p.u., $x_{d i}^{\prime}=0.524$ p.u., \\
& $D_{i}=2.0 \mathrm{~s} /$ rad, $H_{i}=6.5 \mathrm{~s}, T_{d o i}=6.0 \mathrm{~s}$. \\
Generator 2 & $x_{d i}=1.557$ p.u., $x_{d i}^{\prime}=0.503$ p.u., \\
& $D_{i}=2.0 \mathrm{~s} /$ rad, $H_{i}=8.5 \mathrm{~s}, T_{d o i}=5.14 \mathrm{~s}$. \\
Generator 3 & $x_{d i}=1.22$ p.u., $x_{d i}^{\prime}=0.404$ p.u., \\
& $D_{i}=2.0 \mathrm{~s} /$ rad, $H_{i}=7.3 \mathrm{~s}, T_{d o i}=4.84 \mathrm{~s}$. \\
Generator 4 & $x_{d i}=1.432$ p.u., $x_{d i}^{\prime}=0.420$ p.u., \\
SVC & $D_{i}=2.0 \mathrm{~s} /$ rad $, H_{i}=4.5 \mathrm{~s}, T_{d o i}=5.03 \mathrm{~s}$. \\
DC supplementary & $K=100, T_{1}=0.045, T_{0}=0.01$ \\
controller & $K_{D}=110, T_{1 D}=0.03, T_{2 D}=0.02$ \\
\hline
\end{tabular}

Case 2. Fault occurs at bus $5, \lambda=0$, and increase Load 1 by $60 \mathrm{MW}$.

Case 3. Fault occurs at the centre of transmission line, $\lambda=$ 0.5 , and increase Load 2 by $60 \mathrm{MW}$.

Case 4. Fault occurs near bus $6, \lambda=0.9$, and increase both of Load 1 and Load 2 by 50 MW.

Figure 6 depicts the power angles and terminal voltages in Case 1 by adopting decentralized control and WRDCC, respectively.

It can be seen from Figure 6 that, under WRDCC, the controller coordinates FACTS and DC supplementary controller to increase damping, and power angle and terminal voltage can be rapidly stabilized with the sudden load increase. Under decentralized control, however, the stabilization of power angle and terminal voltage takes quite a long period due to the lack of damping.

Figures 7 9 depict the power angles and DC power control performance in Cases $2 \sim 4$ by adopting the decentralized controller and decentralized coordinated controller, respectively. Since the three-phase short circuit fault occurring in the transmission line between buses 5 and 6 makes the greatest effect on Generator 1, it is thus picked as an example to simulate the transient stability performance in different cases.

It can be observed from Figures 7 9 that, under decentralized control, the power angle can be stabilized after several oscillation cycles, which may lead to low-frequency oscillations due to the long period of oscillation, among which, in Case 2, the fault occurs nearest to Generator 1; thus oscillation is the most severe. Under WRDCC, the controller coordinates FACTS and DC supplementary controller to increase damping such that system operates under a strong damping mode, and even the power angles of Generator 1 can be rapidly stabilized. Moreover, under decentralized control, the oscillation amplitude of DC transmission line power is quite large, which may lead to oscillation once more. This can be solved by utilizing the decentralized coordinated controller. 


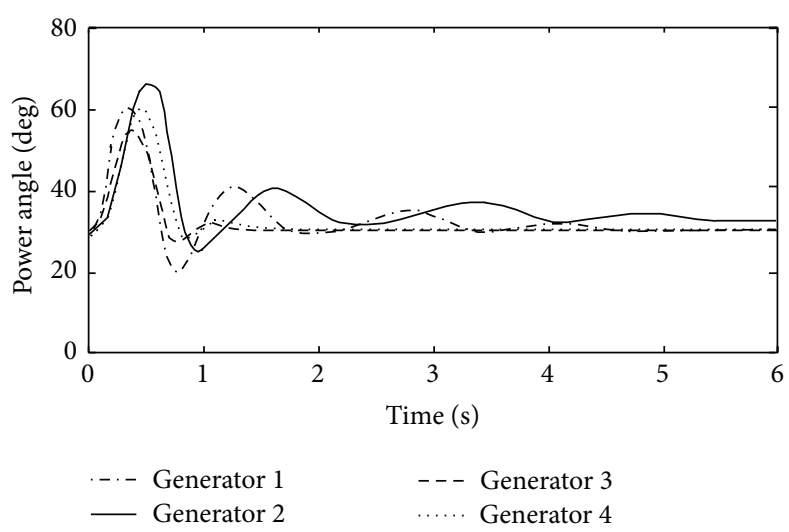

(a) Power angles under decentralized control

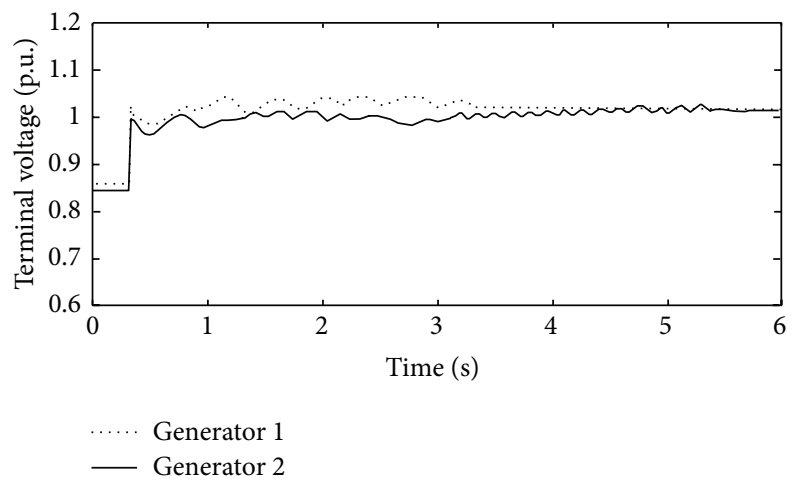

(c) Terminal voltages under decentralized control

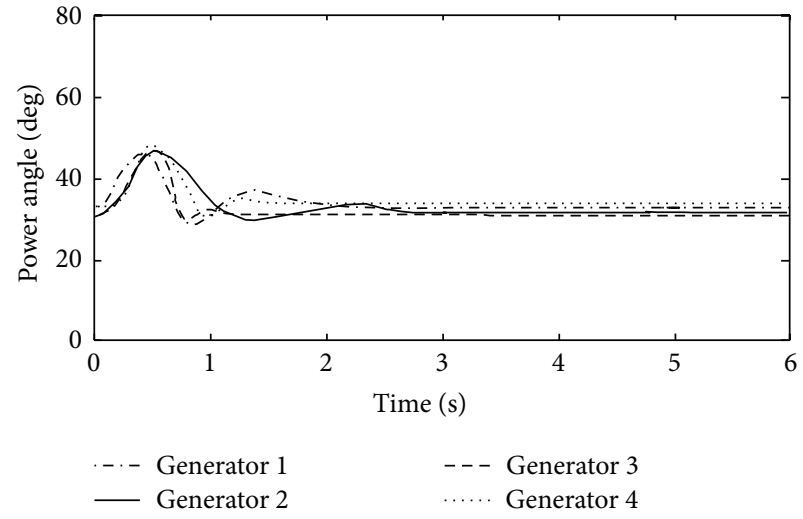

(b) Power angles under WRDCC

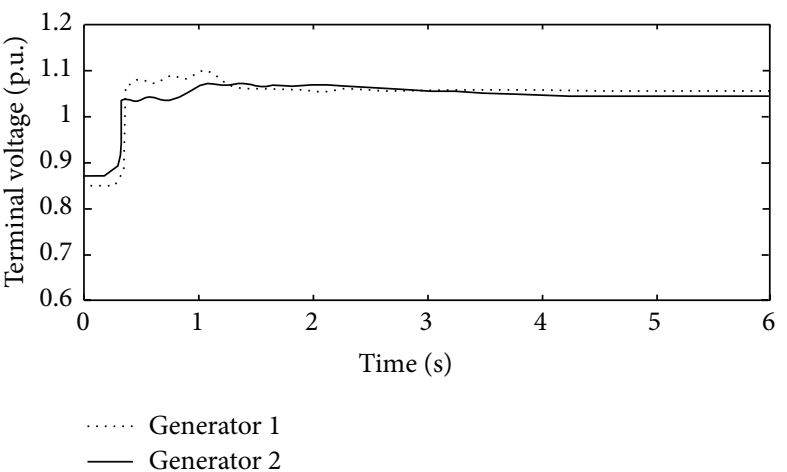

(d) Terminal voltages under WRDCC

Figure 6: Power angles and terminal voltages in Case 1.

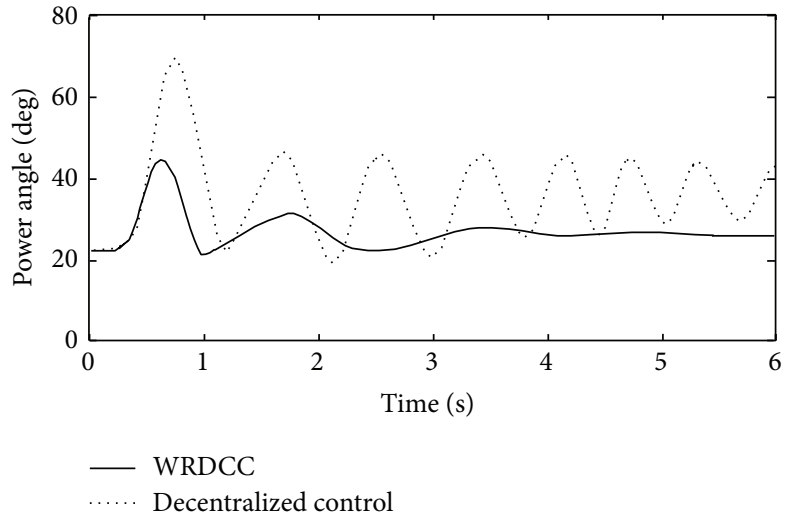

(a) Power angles of Generator 1

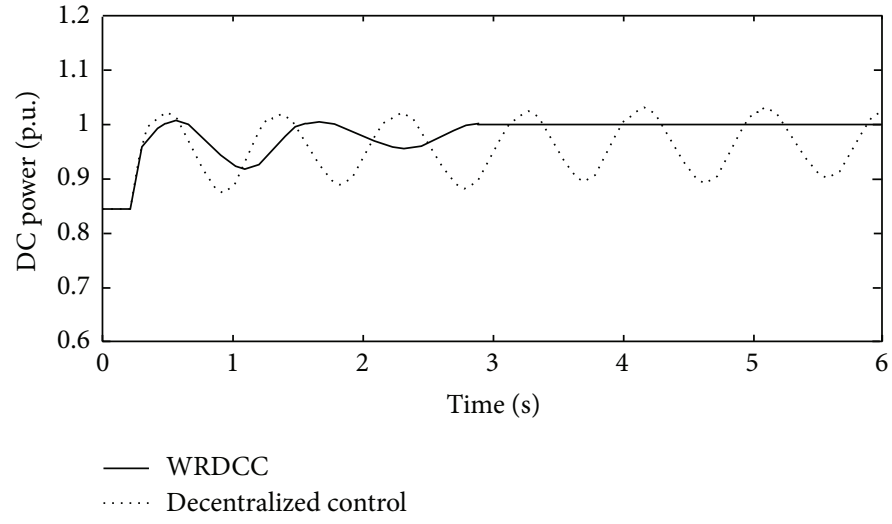

(b) DC power control performance

FIGURE 7: Power angle and DC power control performance in Case 2.

\section{Conclusions}

This study investigates the WRDCC strategy that coordinates DC supplementary controllers, FACTS devices, and PSS in HVDC transmission systems and proposes a robust coordinated control algorithm applicable to various operating conditions. By introducing the polytopic model, the closedloop controller system can operate under strong damping mode in virtue of the stability criterion based on damping ratio. Simulation results in a two-area, four-machine HVDC system equipped with a DC supplementary controller and an SVC demonstrate that, under four different operating 


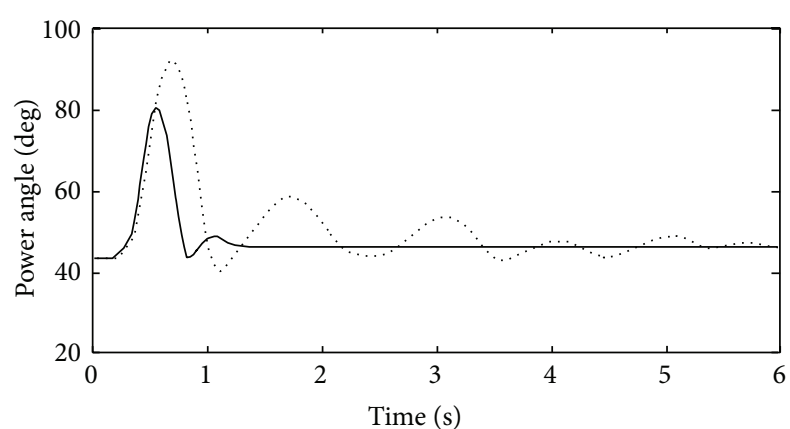

— WRDCC

..... Decentralized control

(a) Power angles of Generator 1

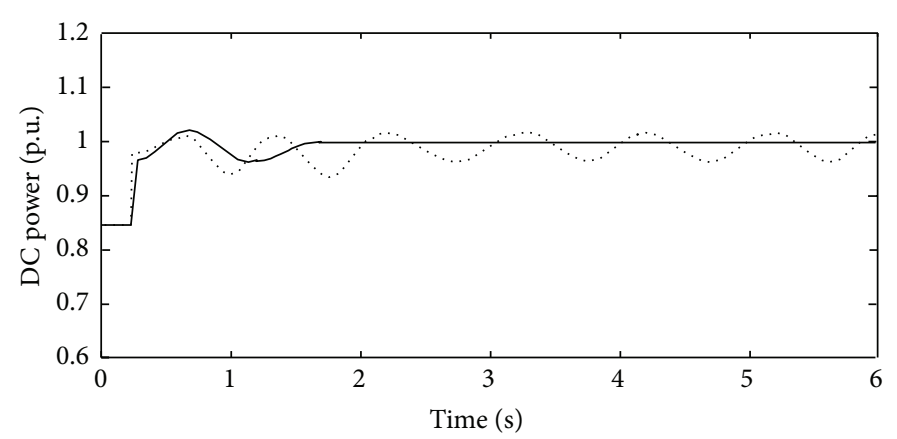

- WRDCC

..... Decentralized control

(b) DC power control performance

Figure 8: Power angle and DC power control performance in Case 3.

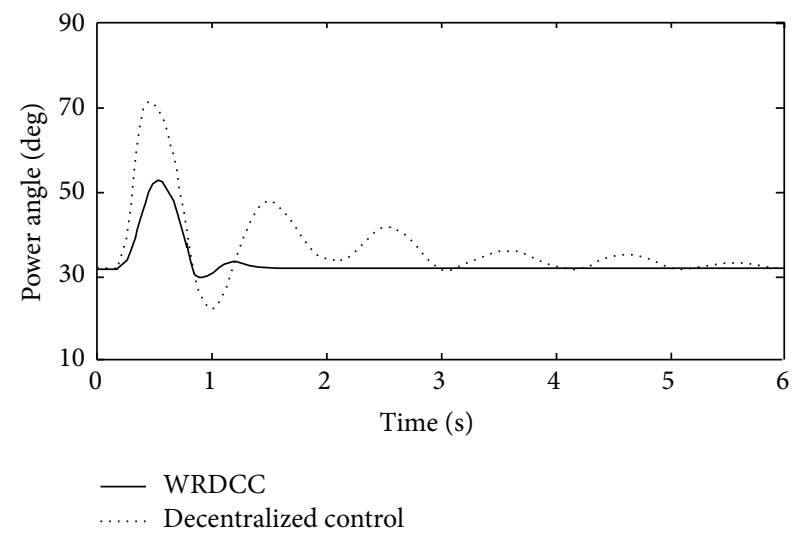

(a) Power angles of Generator 1

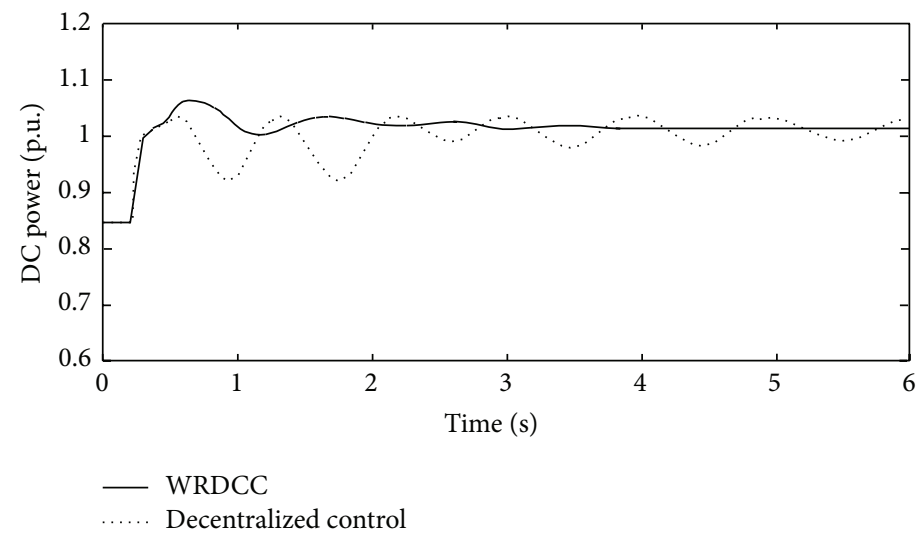

(b) DC power control performance

FIGURE 9: Power angle and DC power control performance in Case 4.

cases, the designed decentralized coordinated controller can increase system damping, rapidly stabilize the power angle and DC power, and maintain good terminal voltage.

\section{Conflict of Interests}

The authors declare that there is no conflict of interests regarding the publication of this paper.

\section{Acknowledgments}

Project was supported by the National Natural Science Foundation of China (51207144); project was supported by State Grid Corporation of China, Fundamental and Forecast Projects (XT71-14-007).

\section{References}

[1] M. Klein, G. J. Rogers, and P. Kundur, "A fundamental study of inter-area oscillations in power systems," IEEE Transactions on Power Systems, vol. 6, no. 3, pp. 914-921, 1991.
[2] B. Chaudhuri, R. Majumder, and B. C. Pal, "Application of multiple-model adaptive control strategy for robust damping of interarea oscillations in power system," IEEE Transactions on Control Systems Technology, vol. 12, no. 5, pp. 727-736, 2004.

[3] R. L. Cresap, D. N. Scott, W. A. Mittelstadt, and C. W. Taylor, "Operating experience with modulation of the pacific HVDC intertie," IEEE Transactions on Power Apparatus and Systems, vol. 97, no. 4, pp. 1053-1059, 1978.

[4] D. E. Martin, W. K. Wong, D. L. Dickmander, R. L. Lee, and D. J. Melvold, "Increasing WSCC power system performance with modulation controls on the Intermountain Power Project HVDC system," IEEE Transactions on Power Delivery, vol. 7, no. 3, pp. 1634-1642, 1992.

[5] Y. Huang and Z. Xu, "HVDC supplementary controller based on synchronized phasor measurement units," in Proceedings of the IEEE Power Systems Conference and Exposition, pp. 668-672, October 2004.

[6] X. M. Mao, Y. Zhang, L. Guan, X. C. Wu, and N. Zhang, "Improv ing power system dynamic performance using wide-area highvoltage direct current damping control," IET Generation, Transmission and Distribution, vol. 2, no. 2, pp. 245-251, 2008.

[7] M. E. Aboul-Ela, A. A. Sallam, J. D. McCalley, and A. A. Fouad, "Damping controller design for power system oscillations using 
global signals," IEEE Transactions on Power Systems, vol. 11, no. 2, pp. 767-773, 1996.

[8] C. Jakpattanajit, N. Hoonchareon, and A. Yokoyama, "A new coordinated control scheme of PSS and FACT devices for improving power system oscillations in multi-machine system," in Proceedings of the International Conference on Power System Technology: Technological Innovations Making Power Grid Smarter (POWERCON '10), pp. 1-8, Hangzhou, China, October 2010.

[9] D. D. Simfukwe, B. C. Pal, R. A. Jabr, and N. Martins, "Robust and low-order design of flexible AC transmission systems and power system stabilisers for oscillation damping," IET Generation, Transmission \& Distribution, vol. 6, no. 5, pp. 445-452, 2012.

[10] S. Boyd, L. El Ghaoui, E. Feron, and V. Balakrishnan, Linear Matrix Inequalities in System and Control Theory, vol. 15 of SIAM Studies in Applied Mathematics, SIAM, Philadelphia, Pa, USA, 1994.

[11] P. Gahinet and A. Nemirovski, LMI Control Toolbox for Use with Matlab, The Mathworks, College Park, Md, USA, 1995.

[12] P. Kundur, Power System Stability and Control, McGraw-Hill, New York, NY, USA, 1994.

[13] K. Zhou, J. C. Doyle, and K. Golver, Robust and Optimal Control, Prentice Hall, 1995.

[14] B. C. Pal, A. H. Coonick, I. M. Jaimoukha, and H. El-Zobaidi, "A linear matrix inequality approach to robust damping control design in power systems with superconducting magnetic energy storage device," IEEE Transactions on Power Systems, vol. 15, no. 1, pp. 356-362, 2000.

[15] M. Chilali and P. Gahinet, " $H_{\infty}$ design with pole placement constraints: an LMI approach," IEEE Transactions on Automatic Control, vol. 41, no. 3, pp. 358-367, 1996.

[16] M. C. de Oliveira, J. C. Geromel, and J. Bernussou, "Design of dynamic output feedback decentralized controllers via a separation procedure," International Journal of Control, vol. 73, no. 5, pp. 371-381, 2000.

[17] R. A. Ramos, L. F. C. Alberto, and N. G. Bretas, "A new methodology for the coordinated design of robust decentralized power system damping controllers," IEEE Transactions on Power Systems, vol. 19, no. 1, pp. 444-454, 2004.

[18] S. Y. Kung and D. W. Lin, "Optimal Hankel-norm model reductions: multivariable systems," IEEE Transactions on Automatic Control, vol. 26, no. 4, pp. 832-852, 1981.

[19] K. J. Astrom and B. Wittenmark, Computer-Controlled Systems -Theory and Design, Tsinghua University Press, Beijing, China, 3rd edition, 2002. 


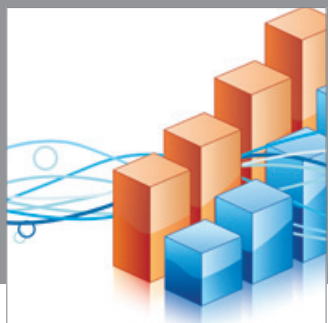

Advances in

Operations Research

mansans

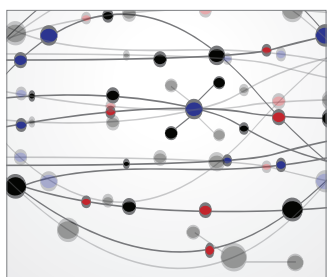

The Scientific World Journal
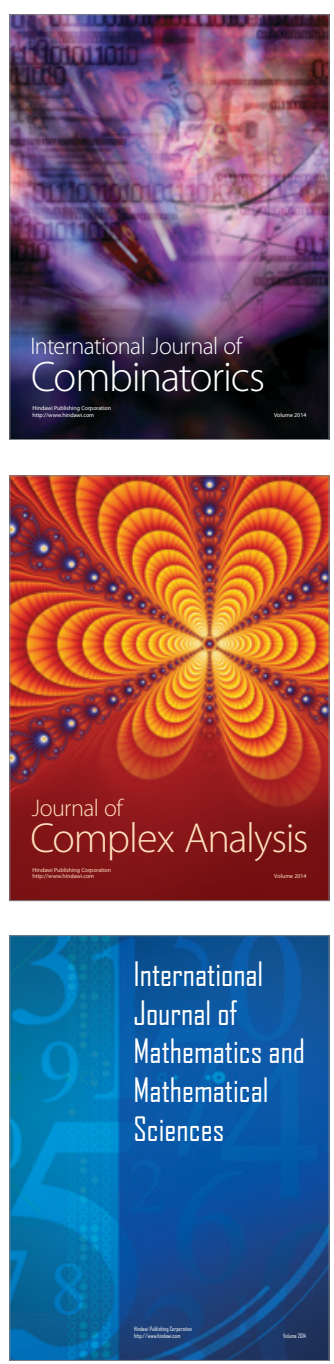
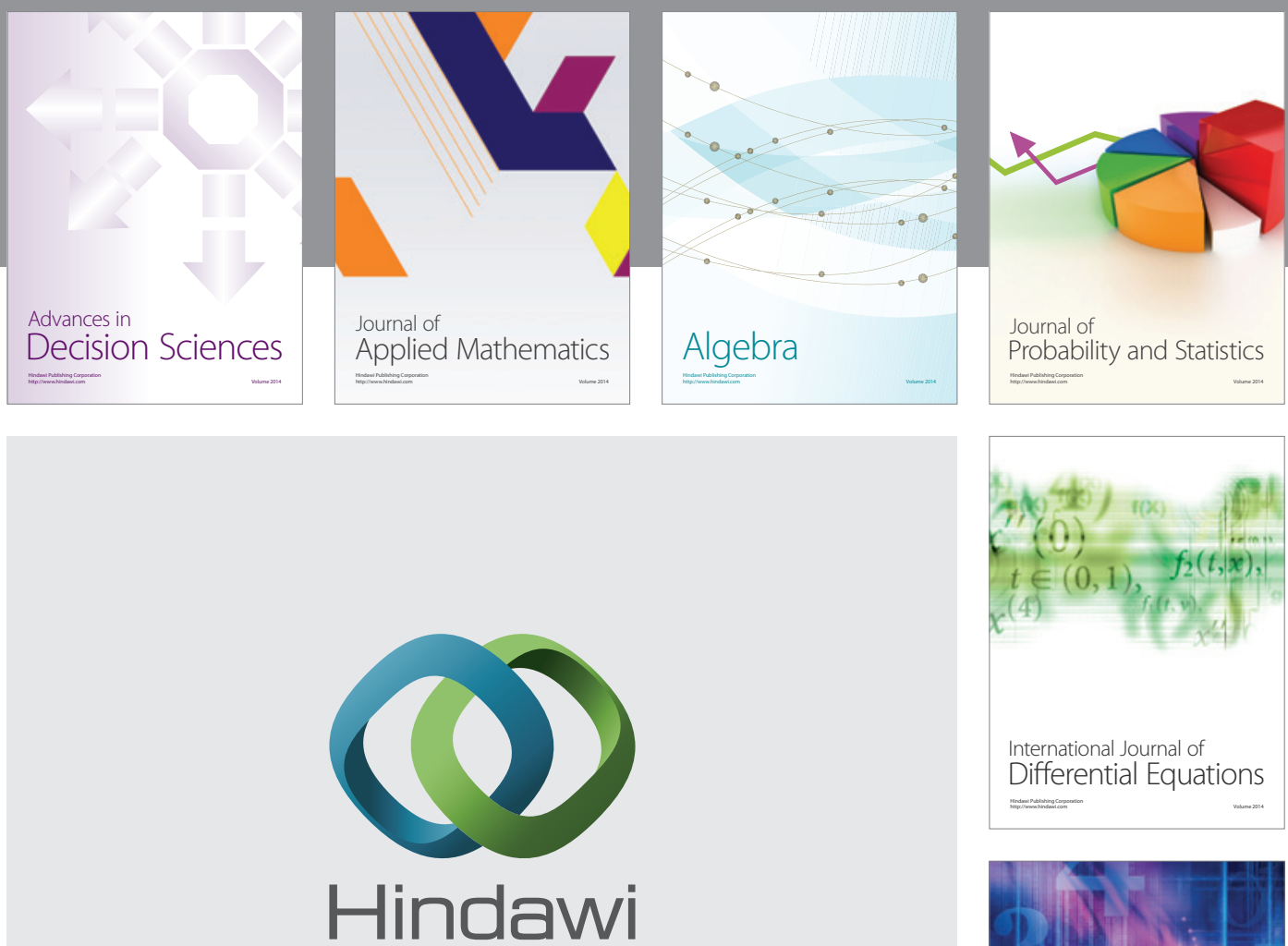

Submit your manuscripts at http://www.hindawi.com
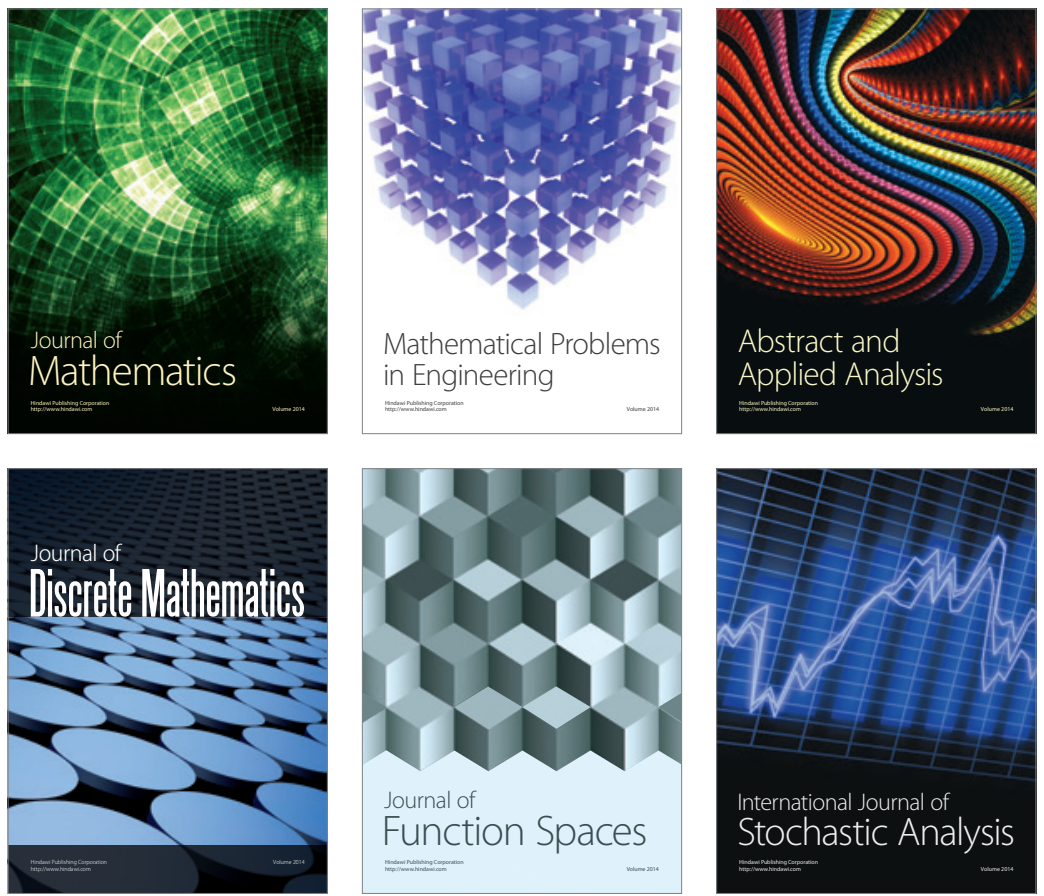

Journal of

Function Spaces

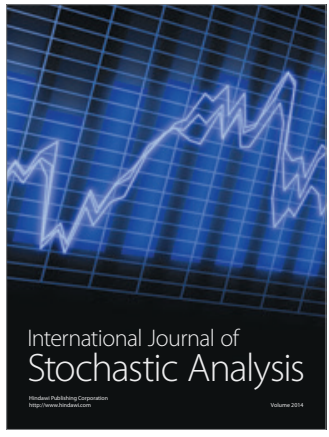

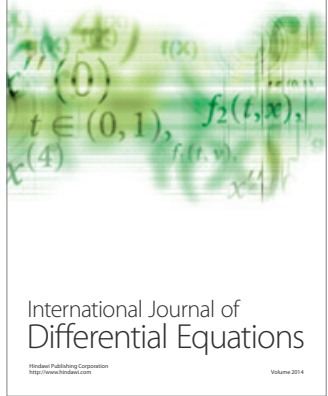
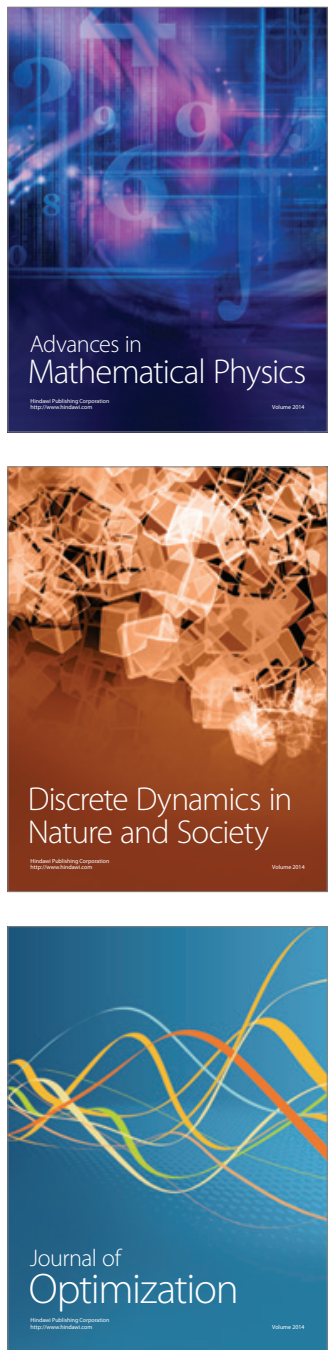